\title{
Microbial Pathogenesis
}

\author{
Ruvin Haidar
}

M.Sc. Medical Microbiology 2nd Year, Dept. of Microbiology, Singhania University, Rajasthan.

\begin{abstract}
For a pathogenic microbe to cause disease in a susceptible host, it must gain access to that host first. The pathogenicity of a microbe is determined by the virulence factors alongside other innate mechanisms. Apart from the initiation of infection, these virulence factors also enable the pathogenic microorganism to survive in the new environment within the susceptible host. They also enable the pathogenic microorganism to invade the host, colonize, and evade the host defense mechanisms. These virulence factors include; invasins, capsules, siderophores, adhesins, enzymes, endotoxins, and exotoxins.
\end{abstract}

Keywords: Pathogenicity factors and Pathological effect on cells

\section{INTRODUCTION}

Pathogenicity refers to the capacity of a pathogen to initiate an infection in the host. For the opportunistic pathogens, they will only initiate infection in the host when the immunity is compromised (Rott \& Goebel, 2011). This capacity to initiate infection is measured by the virulence of the various pathogens ((Johnson, 2017)). For pathogenicity to occur, it is aided by several factors which enable the pathogen to overcome the host barriers and then initiate an infection. These are known as virulence factors. They assist the bacteria to gain access inside the host, start an infection and then escape the host defenses without being destroyed (Bertaccini et al., 2019). These factors include;

Adherence factors: they are used to colonize the mucosal surfaces of the host. An example is pili. In simple terms, they help the pathogen attach to the surface of the target host (Johnson, 2017).

Invasion factors: these are surface substances that enable bacteria to gain access inside the host cells. These factors can be coded on plasmid but they are more often found on the chromosome (Johnson, 2017).
Capsule: offers protection against host immune responses as well as assisting the bacteria to adhere to surfaces. The majority of these capsules are polysaccharides in nature. As compared to other biological macromolecules such as proteins, polysaccharides are less immunogenic and thus protects the bacterial from phagocytosis as they do not elicit an immune response (Rubens, 2014).

Exotoxins: these are the toxins that are produced but the microbes and they do interfere with cellular homeostasis and are also responsible for causing diseases in the host. They are produced in smaller amounts which are unable to stimulate the immune system thus neutralizing the immune responses (Boquet \& Ricci, 2014).

Enzymes: they are active against the host responses and contribute to virulence by destroying the host tissues. Once the host tissues are destroyed they later become susceptible to microbial infections. These enzymes include; proteases, phospholipases, and neuraminidases. They provide nutrition to the microbes by digesting the host cellular materials to smaller components that can be utilized by the microbes (Cole, 2019. 
Adhesins: these are microbial components exposed on the surface and are responsible for adherence in the host tissues. They protect the microbe from host immune responses (Vengadesan \& Narayana, 2011).

\section{Mechanism of microbial pathogenicity Adherence}

This refers to how the microbe gains access to the body. It is a means of the bacteria attaching itself to the host tissues. This needs the involvement of two factors; a receptor and an adhesion. The receptor is a particular carbohydrate or peptide residue on the eukaryotic cell surface. Adhesins on the other hand refer to the macromolecular components on the surface of a bacterial cell that interrelates with the internal receiver of the host (Sastry et al., 2016). The specific adherence of the microbe to the host tissues is observed in;

i. Tissue tropism- in this, particular microbes are notorious for their recognizable prevalence for some tissues as compared to others. For instance, Streptococcus mutans is superabundant in the bacterial plaque in the mouth and absent on the peripheral surface of the tongue whereas Streptococcus salavarius is abundant on the epithelial surface of the tongue and absent on the bacteria plague in the mouth (McCall et al., 2016).

ii. Species specificity- this is where some pathogenic microbes cause diseases in some animal species for example Neisseria gonorrhea are limited only to human beings (Gurtler et al., 2017.

iii. Genetic selectivity among speciescertain strains among a species are genetically resistant to pathogenic microorganisms. For instance, some strains of the pig are resistant to Escherichia coli type K.88 infection.

\section{Invasion}

This is facilitated by extracellular materials which react in the opposition to the host by altering the principal and subordinate immune protection. These substances are known as invasins. Once the host epithelial barrier is penetrated the bacterial pathogen can multiply (Rott \& Goebel, 2011).

\section{Colonization}

In this, there is the multiplication of microbes where the toxins are produced and the normal flora is overcome. They compete with normal flora for space and nutrients. for infection to proceed, an infectious dose should be determined which is the minimum number of microbes required to establish an infection. Some microbes are less contagious requiring a large number while others are more contagious requiring fewer numbers to initiate an infection. During colonization, the host begins to show signs of septicemia (Wilson et al., 2020).

\section{Evasion from host defenses}

These are strategies that are used by the bacterial pathogen to avoid or inactivate host defenses thus ensuring their survival within the host. These include; avoiding phagocyte recognition by producing capsules, utilizing antigenic variation to alter the antigenic structure, production of proteases that enables them to avoid pathogens, producing catalase to prevent digestion of engulfed pathogen and they do mimic host molecules which can cause diseases related damage (Vincent, 2017).

\section{CONCLUSION}

After evasion from the host, the pathogenic microbe causes damage to the host, that is, by causing diseases. The microbe then exits from the host, infects another host and the cycle continues. They are released from the host via various body secretions, coughing among other routes.

Acknowledgement: None

Conflict of Interest: None

Source of Funding: None 


\section{REFERENCES}

1. Bertaccini, A., Oshima, K., Maejima, K., \& Namba, S. (2019). Phytoplasma effectors and pathogenicity factors. Phytoplasmas: Plant Pathogenic Bacteria - III, 17-34. https://doi.org/10.1007/978-981-13-96328_2

2. Boquet, P., \& Ricci, V. (2014). Bacterial exotoxins. Reference Module in Biomedical Sciences. https://doi.org/10.1016/b978-0-12801238-3.00134-3

3. Cole, M. F. (2019). undefined. Unifying Microbial Mechanisms, 187-200. https://doi.org/10.1201/9780429262777-7

4. Gurtler, J. B., Doyle, M. P., \& Kornacki, J. L. (2017). Foodborne pathogens: Virulence factors and host susceptibility. Springer.

5. Johnson, D. I. (2017). Bacterial pathogens and their virulence factors. Springer.

6. Johnson, D. I. (2017). undefined. Springer.

7. McCall, L., Siqueira-Neto, J. L., \& McKerrow, J. H. (2016). undefined. PLOS Pathogens, 12(5), e1005519. https://doi.org/10.1371/journal.ppat.100551 9
8. Rott, R., \& Goebel, W. (2011). Molecular basis of viral and microbial pathogenesis: April 9-11, 1987. Springer.

9. Rubens, C. E. (2014). undefined. Molecular Genetics of Bacterial Pathogenesis, 327339. https://doi.org/10.1128/9781555818340.ch2 1

10. Sastry, A., K, S., \& Janagond, A. (2016). undefined. Essentials of Medical Microbiology, 87-87. https://doi.org/10.5005/jp/books/12637_9

11. Vengadesan, K., \& Narayana, S. V. (2011). undefined. Protein Science, 20(5), 759-772. https://doi.org/10.1002/pro.613

12. Vincent, W. J. (2017). Innate immune cell recruitment and host defense in response to intracellular bacterial infection.

13. Wilson, B. A., Winkler, M., \& Ho, B. T. (2020). undefined. John Wiley \& Sons.

How to cite this article: Ruvin Haidar. Microbial pathogenesis. Int J Health Sci Res. 2021; 11(12): 217-219. DOI: https://doi.org/10.52403 /ijhsr.20211228 